\title{
Intelligent decision-making method for The electric car filling in power station planning and management
}

\author{
Liu Zhijian, Wang Dongdong \\ Kunming university of science and technology,Yunnan,650500,China \\ E-mail:wddkmust@163.com
}

\begin{abstract}
Keywords:decision-making method;electric car;planning and management; GIS;artificial intelligence
\end{abstract}

\begin{abstract}
The present paper is aimed at introducing the author's newly developed decision-making method, known as"intelligent decision-making method for The electric car filling in power station Planning and Management". As we often see, it is a fundamental method to overcome the difficulties brought by great chaos in the process of the urban garbage management, which can only be improved by heightening the effectiveness of decision-making Planning and Management. Therefore this paper has put forward the new method according to the characteristics and existent problems of electric car filling in power station management decision.It is the three levels that form the level model frame of decision-making and determine the decision models respectively. Then the paper has put forward the general frame of the electric car filling in power station management system, with the planned strategic targets and decision analysis about respective links in the system being illustrated. At the same time, it is also necessary to integrate the artificial intelligence with semi-structural and unstructured models of general planning through KBS to absorb the plentiful experience and knowledge ac-cumulated for many years from planning experts so as to combine the quantitative calculation with the qualitative analysis to improve the ability of problem solving.In addition, the general decision models for this method are also provided and the process of decision-making and main innovations of this method are also described step by step.
\end{abstract}

\section{Introduction}

There are still many uncertainties for China electric industry development. In 2009 National car industry restructuring and revitalization plan proposed by 2011, formation of 500,000 electric vehicles, plug-in hybrid and ordinary hybrid electric vehicle capacity goals, 4 years have passed, however, haven't been able to achieve this goal. Development of electric vehicle industry current unsatisfactory reasons are:(1)Lacking of support from a national perspective and reality manipulation.(2)Electric vehicle charging for power plant siting is electric-vehicle charging for infrastructure must first solve the problems which is irrational.[1] with the development model for the study of electric vehicle industry in China, using the method of system dynamics, refines the system dynamics model of electric vehicle industry electricity price policy, making the model has some limitations.[2] sets up geographic information, capital costs and operating cost optimization objective function, quantum swarm optimization algorithm is used to study the optimization layout of urban electric vehicle charging stations.[3] the traditional distribution network to study the electric response of high permeability, comparative analysis of electric vehicles and battery-grid interaction between programmes and programmes, studied the micro-grid for optimal planning of power stations in the distributed generation models.[4] established a record and geographical factors and the service radius of two screening methods, used to determine the electric charge-for-power candidate site.[5] charging charging costs investors minimize cost and charging stations as the optimization goal, established a two-stage heuristic algorithm solving the model.

From the above knowledge, the current development model of China's electric vehicle industry has not been determined, so that the electric vehicle's infrastructure planning, especially the electric vehicle charging station planning and location of the existence of a greater risk and uncertainty. 
2. electric vehicle charging station planning management and electric vehicle charging station planning intelligent decision method

\section{1 electric vehicle charging station planning and management}

In the model establishment, the price is too high, the model and the actual situation is different. In order to solve the defects of the method proposed electric vehicle charging station planning and management idea, that is not only study for electric vehicle charging and operation mechanism model of every subsystem in the power plant planning and interaction between the baryon injection system, a comprehensive analysis of electric vehicle charging station planning system, and then build a scientific and reasonable layout of the overall strategy.

\section{2 intelligent decision making method for electric vehicle charging station planning}

\subsubsection{Classification of decision}

The main idea of decision is: the planning and management of the electric vehicle charging station planning decision-making problem is divided into 3 levels, namely, the operational level, tactical level and strategic level. The first two layers belong to the management level, and the third level is the category of planning. At the same time, there are 3 types of decision making problems in each level, which are structured, semi-structured and unstructured.

\subsection{2 master plan}

According to the above level model, in the full investigation, on the basis of the overall planning objectives, the establishment of electric vehicle charging power plant's overall planning framework, see figure 1.

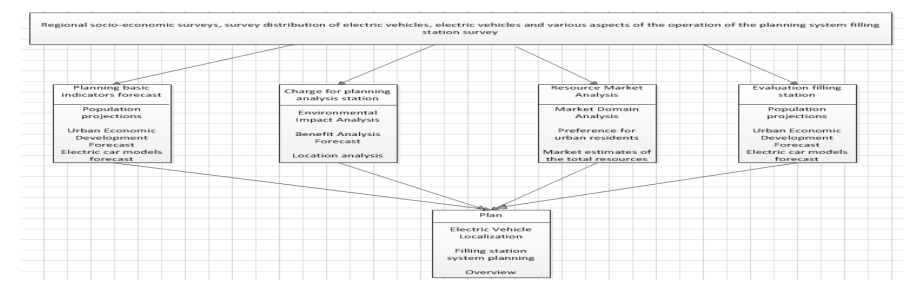

Fig.1 Composition of general planing for electric car filling in power station management

\subsection{3 building decision model}

Firstly,we introduce the artificial intelligence technology to construct the model of the semi structured and unstructured decision problem in the overall planning. Decision making problem extends to more complex and intelligent features of the structured problem, which can be solved by the program design[6]. Therefore, it is not enough to construct a professional model by applying mathematics to construct a professional model. For this purpose, the KBS (Knowledge Based System ) is designed, which is based on the characteristics of the electric vehicle charging station planning system.

Secondly, the model and the GIS are combined to give full play to the role of the auxiliary decision-making. The mathematical model pays attention to the theory and calculation method, and does not pay attention to the research of its form of expression, so the data is not intuitive, the data management and operation are more difficult, and the visual difference is difficult to be used by the users of professional knowledge. In addition, the space expression is very important in the decision of the electric vehicle charging station planning management[7]. They are integrated to establish a space management model, which can make the most of the research object and the most effective information. Management model and GIS integration principle figure 2, the GIS function module is embedded in the management model, the management model and the overall integration of GIS, the establishment of space management model.

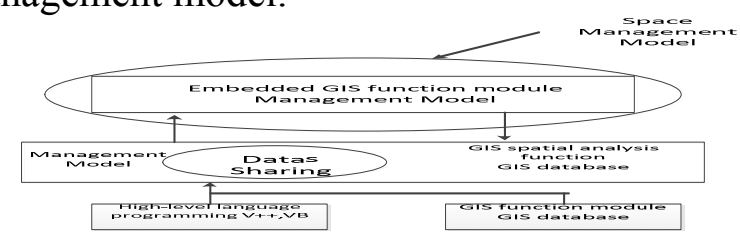

Fig.2 Spatial management model 
The above constitutes the decision-making method based on the idea of planning management, the integration of GIS and the artificial intelligence technology.

\section{3. general model and decision process of intelligent decision making method for electric vehicle charging station planning}

\section{1 general model}

As the basis of the decision method of this paper is to be layered, the problem of high-level problem is solved on the basis of the problem of low level, that is, the solution of the problem is solved. The general model of the decision is given here. Planning (strategic level) decision vector is

$$
\mathrm{PD}=\left(\mathrm{pd}_{1}, p d_{2}, \cdots p d_{n}\right)
$$

$\operatorname{pd}_{i}(i=1, \ldots, n)$ is a planning decision variable, and $\mathrm{N}$ is the number of decision variables.

The sub problem $J$ (management level) decision vector is ${ }^{M D}{ }_{j}(j=1,2, \ldots, m)$.

In general, the decision model of the sub problem can be expressed as

$\max _{\mathrm{MD}} L P_{j}=f_{j}\left(P D, M D_{j}\right), j=1,2, \cdots, m$

The sub problem decision vector $\mathrm{MD}_{\mathrm{j}}$ is a variable, while the planning decision vector $\mathrm{PD}$ is a parameter (constant).

By introducing the decision vector of the strategic level into the sub problem decision model, the relationship between different levels of the model is established.

\section{2 intelligent decision making process for electric vehicle charging station planning}

In summary, the decision-making process is shown in figure 3. 1) by providing the user with the relevant information of the decision making problem, the system is processed and transformed into an example of decision making problem. 2) decision making problems. If the decision problem is solved by using the knowledge base and knowledge base, the model can be used to solve the problem. If the decision problem can be solved by using GIS to create a spatial decision model, the decision problem is transformed into a series of sub problems[8]. 3) comprehensive evaluation of the planning and decision making scheme, and the formation of new domain expert knowledge into the knowledge base for the next decision.

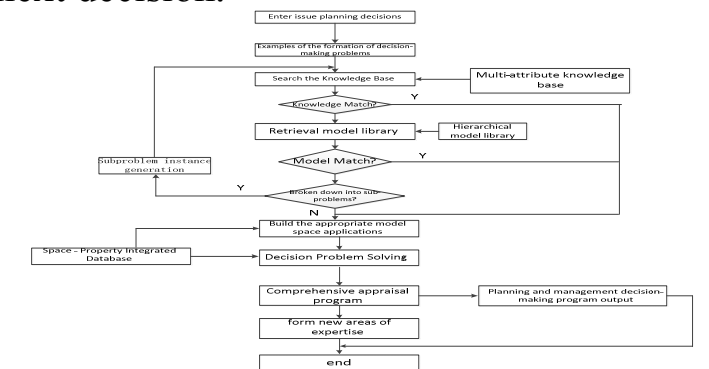

Fig.3 Electric vehicle charging station planning intelligent decision-making process

\section{Conclusions}

In this paper, we use the idea of planning and management to study the location of electric vehicle charging station, combining with GIS and artificial intelligence technology, intelligent decision making method for electric vehicle charging station planning is proposed. This paper presents the ideas of planning and management of electric vehicle charging station for electric vehicle charging station planning system. The model of strategic planning level is reduced by the coordination of sub system. The complexity and difficulty of the model are obtained by using GIS technology and the integrated model. In this method, the design of KBS knowledge base is the basis of this method. Therefore, it is necessary to design the knowledge base of KBS, the specific circumstances of the region as the basis, have the choice to learn from other advanced areas, the national planning and management experience and mode, the formation of its own characteristics of the KBS knowledge base, decision-making will have better intelligence.

This paper provides a theoretical basis for the realization of intelligent decision-making system 
for electric vehicle charging station planning.

\section{References}

[1] Shi Shuya. Research on development model of electric vehicle industry based on system dynamics [D]. Wuhan University of Technology, 2010,1-87.

[2] Zhang Wei, Liu Zifa, Wang Zeli. Optimal layout of urban electric vehicle charging station based on quantum particle swarm optimization [J].Proceedings of the CSEE,2012, 32 (22): 39-42.

[3], Zhao Rongxiang, Yang Huan. The response strategy for high permeability of electric vehicles and the optimal planning of power station [J]. power automation equipment, 2012,32 (9): 7-13.

[4] Zhipeng, Wen Fu Shuan, Xue Yu Sheng, Xin Jian Bo. Electric vehicle charging stations in the optimal siting and sizing [J]. Automation of electric power systems, 2012, 36 (3): 54-59.

[5] Ren Yulong, Shi Lefeng 430071. Electric vehicle charging station optimal distribution and scale [J]. Automation of electric power systems, 2012, 35 (14): 53-57.

[6]CHEN Wenwei.Decision support systems course [M].Beijing:Tsinghua University Press,2004: 117-119.

[7]WU Xincai.Design and principle of GIS[M].Beijing: Publishing House of Electronics Industry,2002: 10-12.

[8]HUANG Tiyun.Intelligence decision support systems[M].Beijing:Publishing House of Electronics Industry, 2001: 159 -160. 